\title{
Early detection and intervention evaluation for people at risk of psychosis: multisite randomised controlled trial
} intervention $^{2}$, Suzanne L K Stewart trial manager ${ }^{1}$, Max Birchwood professor of youth mental health ${ }^{3}$, David Fowler professor of social psychiatry ${ }^{4}$, Andrew I Gumley professor of psychological therapy ${ }^{5}$, Peter B Jones professor of psychiatry ${ }^{6}$, Richard $\mathrm{P}$ Bentall professor of clinical psychology ${ }^{7}$, Shôn W Lewis professor of psychiatry ${ }^{8}$, Graham K Murray senior clinical research associate ${ }^{6}$, Paul Patterson project manager ${ }^{9}$, Kat Brunet research clinical psychologist ${ }^{3}$, Jennie Conroy research cognitive therapist $^{10}$, Sophie Parker research clinical psychologist ${ }^{2}$, Tony Reilly research cognitive therapist $^{11}$, Rory Byrne service user researcher ${ }^{2}$, Linda M Davies professor of health economics ${ }^{12}$, Graham Dunn professor of biostatistics ${ }^{12}$

\footnotetext{
${ }^{1}$ School of Psychological Sciences, University of Manchester, Manchester M13 9PL, UK; ${ }^{2}$ Greater Manchester West Mental Health NHS Foundation Trust, Manchester; ${ }^{3}$ School of Psychology, University of Birmingham, Birmingham, UK; ${ }^{4}$ School of Medicine, Health Policy, and Practice, University of East Anglia, Norwich, UK; ${ }^{5}$ Institute of Health and Wellbeing, University of Glasgow, Glasgow, UK; ${ }^{6}$ Department of Psychiatry, University of Cambridge, Cambridge, UK; ${ }^{7}$ Institute of Psychology, Health and Society, University of Liverpool, Liverpool, UK; ${ }^{8}$ Mental Health and Neurodegeneration Research Group, School of Medicine, University of Manchester; ${ }^{9}$ Early Detection and Intervention Team, Birmingham and Solihull Mental Health NHS Trust, Birmingham; ${ }^{10} \mathrm{Cambridgeshire} \mathrm{and} \mathrm{Peterborough} \mathrm{NHS} \mathrm{Foundation} \mathrm{Trust,} \mathrm{Cambridge;}{ }^{11}$ Early Intervention Team, Norfolk and Waveney Mental Health NHS Trust, Norwich, UK; ${ }^{2}$ Health Sciences Research Group, School of Medicine, University of Manchester
}

\begin{abstract}
Objective To determine whether cognitive therapy is effective in preventing the worsening of emerging psychotic symptoms experienced by help seeking young people deemed to be at risk for serious conditions such as schizophrenia.

Design Multisite single blind randomised controlled trial.

Setting Diverse services at five UK sites.

Participants 288 participants aged 14-35 years (mean 20.74, SD 4.34 years) at high risk of psychosis: 144 were assigned to cognitive therapy plus monitoring of mental state and 144 to monitoring of mental state only. Participants were followed-up for a minimum of 12 months and a maximum of 24 months.
\end{abstract}

Intervention Cognitive therapy (up to 26 (mean 9.1) sessions over six months) plus monitoring of mental state compared with monitoring of mental state only.

Main outcome measures Primary outcome was scores on the comprehensive assessment of at risk mental states (CAARMS), which provides a dichotomous transition to psychosis score and ordinal scores for severity of psychotic symptoms and distress. Secondary outcomes included emotional dysfunction and quality of life.

Results Transition to psychosis based on intention to treat was analysed using discrete time survival models. Overall, the prevalence of transition was lower than expected (23/288; $8 \%$ ), with no significant difference between the two groups (proportional odds ratio $0.73,95 \%$ confidence interval 0.32 to 1.68). Changes in severity of symptoms and distress, as well as secondary outcomes, were analysed using random effects regression (analysis of covariance) adjusted for site and baseline symptoms. Distress from psychotic symptoms did not differ (estimated difference at 12 months $-3.00,95 \%$ confidence interval -6.95 to 0.94 ) but their severity was significantly reduced in the group assigned to cognitive therapy (estimated between group effect size at 12 months $-3.67,-6.71$ to $-0.64, \mathrm{P}=0.018$ ).

Conclusions Cognitive therapy plus monitoring did not significantly reduce transition to psychosis or symptom related distress but reduced the severity of psychotic symptoms in young people at high risk. Most participants in both groups improved over time. The results have important implications for the at risk mental state concept. 
Trial registration Current Controlled Trials ISRCTN56283883.

\section{Introduction}

Reliable and valid criteria are available to identify help seeking people in diverse settings who are at high risk of imminently developing schizophrenia and related psychoses. Researchers developed operational criteria to identify three subgroups possessing an "at risk mental state" for psychosis. . Two subgroups specify state risk factors, defined by the presence of either transient psychotic symptoms, called brief limited intermittent psychotic symptoms, or attenuated (subclinical) psychotic symptoms. The other subgroup comprises trait plus state risk factors, operationally defined by the presence of diminished functioning plus either a first degree relative with a history of psychosis or a pre-existing schizotypal personality disorder. All subgroups are within a specified age range known to be at greatest risk for the onset of psychosis, and all participants in studies to date who are in an at risk mental state have been help seeking, which effectively means this is part of the criteria.

Effective interventions to prevent or delay this transition are needed because of the significant personal, social, and financial costs associated with the development of psychosis. To date six randomised controlled trials have reported findings on outcomes associated with antipsychotic drugs, omega- 3 polyunsaturated fatty acids, and psychological interventions; each using similar operational definitions of at risk mental state. These studies (table $1 \Downarrow$ ) were carried out in Australia, ${ }^{23}$ North America, ${ }^{45}$ the United Kingdom, ${ }^{67}$ and Austria. ${ }^{8}$

We have argued ${ }^{9}$ that cognitive therapy may be well suited to patients who are in an at risk mental state since its efficacy has been shown in acute ${ }^{10}$ and chronic, persistent psychotic symptoms ${ }^{11}{ }^{12}$ as well as in the prevention of relapse ${ }^{13}$ and emotional disorders. ${ }^{14}$ The current multisite, randomised controlled trial was designed as a definitive test of the hypothesis that cognitive therapy would prevent transition to psychosis in a population who were in an at risk mental state, and would reduce the severity of and distress associated with psychotic symptoms. We also hypothesised that cognitive therapy would reduce emotional dysfunction and improve quality of life.

\section{Methods}

We carried out a multisite randomised controlled single blind (rater) trial comparing cognitive therapy plus monitoring of mental state with monitoring of mental state only (control).

We assessed entry criteria using the comprehensive assessment of the at risk mental state (CAARMS), which is specifically designed for the assessment of people in the category of at risk mental states. ${ }^{15}$ The entry routes consist of brief limited intermittent psychotic symptoms, attenuated psychotic symptoms, or state plus trait factors. All candidates were aged between 14 and 35 and seeking help for symptoms. Exclusion criteria were current or previous receipt of antipsychotic drugs, moderate to severe learning disability, organic impairment, and insufficient fluency in English. The five sites involved were Manchester, Birmingham/Worcestershire, Glasgow, Cambridgeshire, and Norfolk. Participants were predominantly identified by health professionals working within diverse agencies within primary and secondary care settings. Table $2 \Downarrow$ shows the referral sources for the trial participants. Our ascertainment strategy was to make services familiar with our entry criteria and to liaise on a regular basis; no systematic screening of service populations was carried out. Because participants were required to be help seeking, we also emphasised the potential benefits of the monitoring alone condition. We excluded 45 potential participants for not help seeking (figure $\downarrow$ ); they were referred as being likely to meet our criteria by a health professional involved in their treatment as usual, but when consulted about participation in the study these individuals made it clear that they were not seeking help.

\section{Interventions}

Participants were randomised to monitoring of mental state only (control) or to cognitive therapy plus monitoring of mental state. Both conditions were in addition to treatment as usual, which will have been highly variable and dependent on local service configurations and specific source of referral to the trial. In an attempt to control for this variation we stratified randomisation by site.

\section{Monitoring}

All participants received treatment as usual plus regular monitoring (incorporating CAARMS from a research assistant), which represents an enhancement over routine care since it aimed to provide warm, empathic, and non-judgmental face to face contact and supportive listening; signposting to appropriate local services for unmet needs and crisis management when required (usually by referral to a local crisis team, early intervention service, or psychiatric liaison within emergency departments). Monitoring ensured that all participants had a general practitioner with whom they were encouraged to stay in regular contact and a personalised "crisis card," which provided contact details for local sources of help in a psychiatric emergency. Staff involved in monitoring and those delivering cognitive therapy did not overlap.

\section{Monitoring plus cognitive therapy}

In addition to the monitoring component, participants allocated to the therapy arm of the trial received cognitive therapy based on our specific cognitive model. ${ }^{16}$ Sessions were offered on a weekly basis for up to a maximum of 26 weeks, plus up to four booster sessions in the subsequent six months. For many participants, however, it was expected that the duration of cognitive therapy would be shorter than this, since shared goals may be reached earlier; in previous cognitive therapy trials based on our manual, the mean number of sessions was $11^{6}$ and $12 .{ }^{5}$ Cognitive therapy requires an individualised, problem oriented approach and incorporates a process of assessment and formulation, which is manualised. The specific interventions depend on individual goals and formulations, but the range of permissible interventions is described in our manual. ${ }^{17}$ Key ingredients of the approach are the development of a problem and goal list, early formulation (both longitudinal and maintenance), a focus on normalising psychotic-like experiences, and an active therapy stance utilising behavioural experiments and evaluation of appraisals. Fidelity to the treatment protocol was ensured by regular supervision of the therapists and assessed by rating recordings of sessions using a revised version of the cognitive therapy scale ${ }^{18}$ and the cognitive therapy for at risk populations adherence scale. ${ }^{19}$

Staff in the study sites were trained initially, and therapy supervision was provided by weekly meetings between therapists and investigators. Cognitive therapy sessions were taped with the participant's consent so that participants could be asked to listen to the tapes as part of their homework and to assist supervision. During the course of the trial a sample of 80 tapes was rated according to the cognitive therapy scale-revised ${ }^{18}$ and the cognitive therapy for at risk populations adherence scale ${ }^{19}$ 
to ensure rigorous adherence to the protocol throughout the duration of the trial. These tapes were drawn from both early and late phases of therapy and included participants from each year of recruitment.

\section{Cognitive therapy}

Thirteen therapists delivered the cognitive therapy. The number of participants treated by each ranged between 2 and 35: mean 11 (SD 9). Manchester had five therapists,

Birmingham/Worcestershire and Glasgow each had three, and Cambridgeshire and Norfolk each had one. Eight were clinical psychologists (doctoral level) and five were nurses with an additional specialist qualification in cognitive therapy. All received additional training associated with the trial manual and received weekly individual supervision and monthly peer supervision.

\section{Outcomes}

The primary outcome measures were transition to psychosis, severity of psychotic symptoms, and distress caused by psychotic symptoms. Each of these was measured using the CAARMS,${ }^{15}$ which provided the data for our primary analyses examining the effect on time to transition to psychosis, the reduction in the severity of psychotic symptoms, and the reduction in distress caused by psychotic symptoms. The primary endpoint for each of these was 12 months, since follow-up was planned on all participants to this point. Transition to psychosis was operationally defined on the CAARMS using the recommended criteria of a global rating scale score of 6 on either unusual thought content, non-bizarre ideas, or disorganised speech, or 5-6 on perceptual abnormalities, with an associated frequency score of 4-6, and with these experiences lasting longer than one week. CAARMS symptom severity was operationalised as the summed scores of the product of global rating scale score (0-6) and frequency $(0-6)$ of the four subscales. Distress was operationalised as the average distress score (0-100) of the four subscales. An adjudication panel was established to ensure that judgments on transition were reliable and valid; investigators who were blind to treatment status (RPB, AIG, SWL, APM, PP) were asked whether they agreed with the CAARMS ratings of those participants identified as making transition on the basis of detailed information from vignettes describing the experiences of the participants. All such ratings achieved consensus. Inter-rater reliability of the CAARMS ratings was assessed regularly (on eight occasions) over the duration of the trial, using both video and role play assessments, with all trial raters participating; intraclass correlation coefficients indicated good reliability between raters: mean 0.90 (SD 0.03). The primary outcomes were amended to incorporate the non-dichotomous variables (severity and distress) in addition to transition, following a decision by the independent data monitoring and ethics committee, the trial steering committee, and the representative of the funding body (Medical Research Council); this was taken at the 18 month point, when it became apparent that the combined (blinded) transition rate was much lower than expected (and required by the power calculation).

Secondary outcomes included emotional dysfunction and quality of life. At baseline and each follow-up, we administered the global assessment of functioning, the Beck depression inventory for primary care, ${ }^{20}$ and the social interactions anxiety scale. ${ }^{21}$ At baseline and at $6,12,18$, and 24 months, we additionally administered the Manchester short assessment of quality of life. ${ }^{22}$ The structured clinical interview for the Diagnostic and Statistical Manual of Mental Disorders, fourth edition
$(\mathrm{DSM}-\mathrm{IV})^{23}$ was used at baseline to determine the presence of axis I disorders and at exit from the trial to determine any psychotic diagnoses; we also administered the psychotic disorders module of the structured clinical interview for DSM-IV if a transition event occurred. These data, in combination with the case vignettes, were reviewed by two professors of psychiatry (PBJ, SWL) to establish psychotic diagnoses (consensus was reached in all but one case, in which consensus was established with further input from GKM). The social interactions anxiety scale has a recommended cut-off greater than 36, indicating a probable diagnosis of social anxiety disorder, ${ }^{24}$ and the Beck depression inventory for primary care has a recommended cut-off greater than 3 , indicating a probable diagnosis of major depressive disorder. ${ }^{20}$ We recorded prescriptions of antipsychotic and other psychiatric drugs.

Our earlier trial used a single baseline assessment, and two of 60 participants disclosed after randomisation that they had been having experiences that met criteria for psychosis. Therefore our current trial assessed participants for eligibility across two CAARMS assessments over 2-4 weeks. This ensured, as much as possible, that we could exclude any individual who was experiencing or had experienced psychosis.

All participants receive monthly monitoring of mental state for the first six months after randomisation, as previous studies have shown that this is the period of maximum risk of transition. After that, they were monitored every three months up to two years. Our variable follow-up period meant that participants recruited in the first 14 months of the study (November 2006-December 2007) were planned to receive the full two year follow-up. Participants recruited thereafter were offered steadily reducing follow-up periods, depending on the time of recruitment. The minimum follow-up period was 12 months.

\section{Sample size}

We determined that a two group $\chi^{2}$ test with a 0.05 two sided significance level would have $80 \%$ power to detect the difference between a $15 \%$ transition rate in the cognitive therapy group and a $30 \%$ transition rate in the control group (odds ratio 0.412) when the sample size in each group was 121 . To allow for a dropout rate of up to $25 \%$, we set our recruitment goal at 320 . We made no allowance for possible therapist effects in these calculations. These rates of transition were chosen on the basis of the original small cohort studies and trials, since the more recent (larger) studies, with lower transition rates, were not available when funding was sought.

\section{Randomisation and blinding}

After the second baseline assessment, participants were randomised electronically using OpenCDMS. ${ }^{25}$ The algorithm uses randomised permuted blocks with block sizes of six or eight, after stratification by site and sex. OpenCDMS then sent out email notification of the allocation to the therapists and trial manager. Thus the results of the randomisation were concealed from the assessors and randomisation was independent.

Assessors were blind to treatment condition. Many strategies were used to achieve blind ratings, including research workers not being involved in the randomisation process, therapists being required to consider room use and diary arrangements in the light of potential blind breaks, and patients being reminded by assessors not to talk about treatment allocation. Overall, 67 blind breaks were reported representing $22.2 \%$ of participants. Therefore blinding was successfully maintained in $77.8 \%$ of participants. Fifteen of these 67 blind breaks were in the monitoring alone condition and 52 in the cognitive therapy 
condition. In cases where blinding was broken, another rater assessed the patient for all subsequent assessments or the ratings were discussed with a blind rater and consensus reached (the latter was only carried out if there was a clinical justification not to switch, such as risk considerations or tentative engagement with the trial). This assessment strategy ensured that only a few of about 1900 assessments had their validity threatened by lack of rater blinding.

\section{Statistical analysis}

Analysis was agreed with the data monitoring and ethics committee, and the a priori analysis plan was published. ${ }^{26}$ Analyses were undertaken in Stata (version 11) after completion of endpoint assessments. The primary analysis was by intention to treat.

We analysed transition to psychosis using discrete time survival models to take into account both the discrete timing of the follow-up assessments (that is, grouped survival times at three month intervals) and the random censoring introduced by the shorter follow-up periods for participants recruited towards the end of the trial. This involved use of a logistic regression model that corresponds to Fienberg's continuation odds model for grouped survival times. ${ }^{27}$ Because there were so few events, we made no allowance for any baseline covariates. Changes in symptom severity and distress as rated by CAARMS, as well as secondary outcomes, were analysed using random effects regression (analysis of covariance) models with summed scores as dependent variables, allowing for attrition and the variable follow-up times introduced by the design of the trial. The use of these models allows the analysis of all available data, on the assumption that data are missing at random, ${ }^{28}$ conditional on adjustment for centre and observed baseline scores. The models allowed for linear, quadratic, and cubic trends in symptom scores over time, but only involved testing the treatment by linear trend interaction - that is, based on the assumption that quadratic and cubic trends would be the same for both groups. We report robust standard errors, significance levels, and confidence intervals. We made no adjustments to allow for multiple testing (using the three primary outcomes, for example).

\section{Results}

Recruitment was completed in June 2009. The final sample size was 288 (80 in Manchester, 77 in Birmingham/Worcestershire, 61 in Glasgow, 30 in Cambridgeshire, and 40 in Norfolk), with 144 patients in each trial arm. Table $3 \Downarrow$ presents the characteristics of the whole sample and the baseline balance across the two groups. Overall, $67 \%$ of the sample had at least one DSM-IV diagnosis at study entry. Specifically, those meeting DSM-IV diagnostic criteria for emotional disorders at baseline included 90 with major depressive disorder (33.7\%), 20 with dysthymic disorder (7.5\%), 17 with panic disorder with agoraphobia (6.4\%), 36 with panic disorder without agoraphobia $(13.5 \%), 30$ with social phobia (11.2\%), 29 with specific phobia (10.9\%), 23 with generalised anxiety disorder (8.6\%), 20 with obsessive compulsive disorder (7.5\%), and 6 with post-traumatic stress disorder (2.2\%). More detailed baseline characteristics and demographics for the participants are described elsewhere. ${ }^{26}$

Those allocated to cognitive therapy received a mean of 9.11 (SD 6.69; range 0-26) sessions, each lasting on average one hour. Adherence to cognitive therapy was reasonably good, with only 9 of $144(6.25 \%)$ patients not attending any sessions and $108(75 \%)$ receiving at least four or more sessions. Fidelity to the therapy model was assessed using competency and adherence scales in relation to audio recordings of 80 therapy sessions. In total, $90 \%$ of rated sessions scored over the threshold for competency and $93.3 \%$ met the criteria for therapy that adhered to the manual.

Table $4 \Downarrow$ shows the time to transition event for each group. Ten (6.9\%) patients who received cognitive therapy made CAARMS defined transition to psychosis, whereas $13(9.0 \%)$ who received monitoring alone made CAARMS defined transition to psychosis. The effect of randomised groups on the odds of an event (transition) for each of the eight time intervals was tested simultaneously using a logistic regression model corresponding to Fienberg's continuation odds model for grouped survival times. ${ }^{27}$ This logistic regression resulted in a non-significant treatment effect (continuation odds ratio $0.73,95 \%$ confidence interval 0.32 to $1.68, \mathrm{P}=0.45$ ). The DSM-IV diagnoses of those who made transition were: eight for schizophrenia, five for schizoaffective disorder, three for delusional disorder, three for schizophreniform disorder, three for psychotic disorder not otherwise specified, and one for brief psychotic disorder.

Table $5 \Downarrow$ shows the results of the primary outcome (CAARMS) at the 6,12 , and 24 month endpoints. The estimated variables include a main effect of treatment and a linear effect of treatment by month interaction. Months of treatment had been centred on 12 months (months=0 at 12 months' follow-up) so that the main effect of treatment corresponds to the difference between the two arms determined at 12 months. The treatment by month interaction indicates how the mean outcomes for two treatment arms are diverging over time. For severity of psychotic symptoms, the treatment by time interaction was not significant, but the difference between treatment arms at 12 months (cognitive therapy minus control) was estimated to be -3.67 (95\% confidence interval -6.71 to -0.64 ), which was significant $(\mathrm{P}=0.018)$. The negative sign indicates that participants in the cognitive therapy arm were doing better than the controls. For distress, the treatment by time interaction was not significant and the estimated difference at 12 months was -3.03 ( -6.95 to 0.94; $\mathrm{P}=0.14)$.

Table 5 also shows the results of the secondary outcomes at the 6,12 , and 24 month endpoints. Treatment effects with analysis of covariance estimates after adjustment for centre and baseline scores were non-significant for the global assessment of functioning (estimated difference at 12 months $1.85,-1.34$ to 5.05; $\mathrm{P}=0.26)$, depression $(-0.37,-1.34$ to $0.60 ; \mathrm{P}=0.45)$, social anxiety $(-2.77,-5.98$ to $0.45 ; \mathrm{P}=0.09)$, and quality of life (2.24, -0.60 to $5.08 ; \mathrm{P}=0.12$ ). Note that these analyses are based on data from those participants with non-missing baseline covariate corresponding to the selected outcome. This was a potential source of bias and lack of precision in the case of the Manchester short assessment of quality of life, which had a lot of missing measurements at baseline (see table 3). Two simple modifications were made to the analysis to check whether this might be the case: dropping the baseline covariate to increase the sample size from 141 to 190 , and using a simple mean imputation (by site and sex) to replace the missing baseline values. In both cases the estimated treatment effect at 12 months decreased to about zero ( -0.06 and 0.007 , respectively). Table 5 also shows the rates of participants scoring above the threshold for a likely diagnosis of social anxiety and major depressive disorders at each time point.

To examine the contribution of the exposure to cognitive therapy, instrumental variable regression using the adjusted treatment received algorithm was used to estimate the effects of number of sessions on 12 month outcomes. ${ }^{29}{ }^{30}$ This method is described in detail in the context of dose-response effects in psychotherapy trials. ${ }^{31}$ A regression model was first fitted for the effects of site and randomised group on sessions, and the 
residuals from this model were saved and used as an additional covariate in a second regression model for the effects of sessions on outcome, allowing for site and the baseline value of the outcome under investigation. This two stage procedure allows for missing outcomes assuming that they are missing at random. ${ }^{28}$ The only significant effect of sessions was found to be on severity, as assessed by CAARMS (estimated effect at 12 months -0.782 per session, $95 \%$ confidence interval -1.329 to $-0.234 ; \mathrm{P}=0.005$ ).

Throughout the duration of the trial 14 participants in the cognitive therapy arm were prescribed antipsychotics compared with 13 in the monitoring alone arm.

\section{Discussion}

Although cognitive therapy for young people (aged 14-35) meeting the criteria for being in an at risk mental state did not affect transition to psychosis over 12-24 months, it significantly reduced the frequency and intensity of psychotic experiences. Cognitive therapy did not significantly affect distress related to these psychotic experiences as measured by the CAARMS, nor levels of depression, social anxiety, or satisfaction with life.

\section{Comparison with other studies}

This is the first multisite randomised controlled trial of cognitive therapy for people in an at risk mental state and the largest randomised controlled trial of this population to date. The results are consistent with findings from clinical trials on people in an at risk mental state. Most trials found that severity of psychotic experiences could be reduced over a moderate timeframe, ${ }^{2468}$ such as 12 months, whereas some found that it was not possible to reduce transition to psychosis over such a period. ${ }^{245}$ The exceptions to this were our earlier single site trial and an Austrian trial involving polyunsaturated fatty acids, both of which found a reduction in transition rate at 12 months. These trials were, however, smaller than the present one, which may have led to a type 1 error. A reduction in severity of psychotic symptoms is clearly desirable in young people at risk. The lack of an effect on transition to psychosis is, however, disappointing. In our trial the low rates of transition (6.9\% for cognitive therapy and $9.0 \%$ for monitoring) are consistent with the declining rates of transition observed in populations in an at risk mental state, which were commented on recently, ${ }^{32}$ especially those found in clinical trials (table 1). Our transition rate for cognitive therapy was almost the same as that in our original trial (6\%), although the low rate observed in the control condition brings into question how "at risk" such patients were, and also means that our trial was significantly under-powered to detect a difference in these observed conversion rates. Although we failed to show a statistically significant effect of the intervention we cannot rule out a beneficial effect of the cognitive therapy on transition rate (although it could be argued that the sample size required to show such an effect, and the small effect sizes reported here, would make such an endeavour unfeasible in practical terms and unwarranted in clinical terms). Our secondary outcomes did not show any benefit from active treatment, although anxiety and depression noticeably decreased over time in both groups, suggesting a natural recovery process in operation. Similarly, satisfaction with life improved for both groups over time. It may be possible to affect such outcomes directly with a more targeted intervention that specifically aims to improve depression and social anxiety.

Given that the significant effects on severity resulted in an estimated difference of about 4 points on our scale (an interaction of frequency and intensity, each of which are 0-6), this represents a clinically meaningful improvement (for example, changing from an intensity rating of severe to moderate or moderately severe to mild, with an accompanying change in frequency from daily or several times a day to less than twice a week). Each session was associated with about a 1 point difference in severity, which itself is of less clinical significance, but an accumulation of sessions will lead to clinically significant change.

\section{Strengths and limitations of the study}

Our trial shows methodological rigour in several ways. Large trial sample sizes generally produce smaller effects, and ours is the largest trial to date with a population in an at risk mental state. Our findings, therefore, follow the trend observed in trials of specific psychological therapies, such as cognitive therapy, which have shown that effect sizes are decreased when indices of study quality (such as adequate statistical power and a control condition that is more active than treatment as usual) are controlled for, within cognitive therapy for both depression ${ }^{33}$ and psychosis. ${ }^{34}$ Importantly, we prespecified the primary and secondary outcomes to be analysed, reducing the likelihood of type 1 errors. The use of five sites should ensure generalisability to routine clinical service provision. However, there are some potential difficulties with our trial. The significant finding of a reduction in severity of psychotic experiences as a result of cognitive therapy would become of borderline significance if we were to adjust for multiple testing using a Bonferroni correction (there being three primary outcomes). We excluded 29 participants who had reported meeting the criteria for being in an at risk mental state at first baseline assessment but reported psychotic experiences consistent with transition at the second baseline assessment. This was a cautious manoeuvre to eliminate the possibility that such individuals were under-reporting at baseline and were, therefore, appropriately excluded on the basis of psychosis at first baseline assessment. Alternatively, they could have been incorrectly excluded (that is, were genuinely at risk at first baseline assessment and developed psychosis in the intervening period). If so, we may have excluded those participants at highest risk of immediate transition, which would adversely affect the statistical power of the trial. The inclusion of these 29 would have given an overall transition rate of $18 \%$, which is similar to that found in the recent cohort studies. A significant proportion of the data was missing, which also may introduce the possibility of bias. As the proportions were similar for both groups we attempted to identify whether people had made transition to psychosis when lost to follow-up by way of reports from family doctors of contact with services and prescription of antipsychotics. This showed that only one participant seemed to have made transition, who had been allocated to the monitoring only condition. We did not measure exposure to treatment before study entry (except for antipsychotic drugs), so we are unable to allow for this in our analyses. Given the lower than expected transition rates, our sample used CAARMS criteria with a $30 \%$ reduction in global assessment of functioning score for the family history group only; subsequent versions of the CAARMS have applied a $30 \%$ reduction in global assessment of functioning score for all groups.

\section{Conclusions and implications}

The reduced transition rate as well as the natural course of recovery that was observed for many of the participants in both conditions, is of considerable interest, and could be due to our sampling approach, the effectiveness of an active control condition, or the lack of validity of the concept of being in an 
at risk mental state. It is possible that the need to actively recruit to target using ascertainment strategies in such diverse settings, modified the "risk enriched" status of the sample such that we included people with a lower risk of transition. ${ }^{35}$ However, our recruitment strategy was similar to that in most of the other trials, our entry routes were equivalent to other trials (predominantly through the attenuated symptoms pathway), and our baseline levels of psychotic experiences and functioning (for example, global assessment of functioning scores) were also similar to those of the other trials. It is likely that the active monitoring control included therapeutic aspects (supportive listening, access to crisis care, signposting), which were more beneficial than envisaged, consistent with the suggestion that benign and simple interventions are effective earlier in the emergence of psychotic disorders, implicit within the concept of a clinical staging model.$^{36}$ It is also possible that the discrepancies in transition rates between the clinical trials with active control conditions (range $0-22 \%$ over 12 months, table 1) and the large recent cohort studies ${ }^{37} 38$ (range $14-22 \%$ over 12 months) may be influenced by factors such as participation in a trial and the strength of therapeutic benefits of active control conditions. This would be consistent with the high engagement rates and low dropout rates found in the trials with psychosocial interventions (table 1).

Our observations also suggest that a review of the "ultra high risk" strategy, in particular the influence of community ascertainment strategies on risk or protective factors for psychosis and of natural recovery processes in adolescence and early adulthood, is required. In relation to the recovery in early adulthood, epidemiological studies of psychotic-like experiences suggest an increase and decrease in such experiences in late adolescence, ${ }^{39}{ }^{40}$ which may impinge on ascertainment strategies for at risk mental states, especially given that about $80 \%$ of such participants meet the criteria on the basis of such attenuated symptoms. Even in the recent cohort studies, the 12 month transition rates were considerably lower than the $50 \%$ at 12 months from the original progenitor cohort study. ${ }^{1}$ Taken together, these findings have important implications for the proposal to incorporate a psychosis risk syndrome in DSM-V, raising questions about its utility as a predictive concept and also about the likely cost-benefit ratio of providing treatment for this group. ${ }^{41}$

The at risk mental state paradigm has been of great benefit in developing thinking about prevention, but these data suggest it needs further research and refinement, particularly in light of the findings on the declining transition rates and their link with community sampling strategies that are required to obtain large samples. The natural ebb and flow of psychotic experiences and co-occurring affective symptoms within adolescence, ${ }^{39}{ }^{40}$ as well as the findings that psychotic experiences are often reported by patients with both affective and anxiety disorders ${ }^{42}$ and that the persistence of psychotic experiences is linked with increased levels of affective symptoms, ${ }^{43}$ have led to suggestions that depression and anxiety should be considered as necessary conditions for the onset of psychosis. ${ }^{44}$ If the criteria for at risk mental state were revised to incorporate such affective disturbances, this could identify a higher risk sample; this is in keeping with the findings of recent cohort studies that have examined predictors of transition. ${ }^{37} 38$ The affective disturbances within our population may trigger help seeking behaviour but it may also be that increasing emotional dysregulation is intrinsically linked to the acceleration of psychotic experiences, possibly through negative appraisal (as threat related or loss related), and would, therefore, predict transition to or an increase in severity of psychosis. This requires further research on the process of transition to psychosis itself. If this turns out to be the case, then simple and benign interventions that target affective disturbances (such as behavioural activation, short term cognitive therapy, interpersonal psychotherapy, and antidepressant or anxiolytic drugs), together with a focus on how clients appraise these experiences and regulate affect, could offer a more powerful method of reducing distress and transition. This would be in keeping with the findings in our trial, since it is clear that both groups improved significantly in terms of psychotic experiences, distress, anxiety, and depression. It is also possible that the therapeutic effects of our monitoring control were greater than expected, since it provided regular contact with non-judgmental, warm, empathic, and accepting individuals who provided a non-stigmatising, non-catastrophising reaction to disclosures of unusual experiences and beliefs, which may have been beneficial. This would also be consistent with recent findings that brief, relatively simple, psychological interventions that target anxiety processes (worry) have a significant effect on psychotic experiences (persecutory ideation) in people with psychosis. ${ }^{45}$ In summary, it is important that future research examines the developmental processes involved in both transition to psychosis and resilience within populations in an at risk mental state, but it seems highly premature to introduce a diagnostic category into DSM-V on the basis of risk of psychosis, given the low transition rate and high potential for natural recovery. This population is clearly help seeking and distressed, with the majority meeting criteria for a diagnosable emotional disorder. ${ }^{46}{ }^{47}$ Therefore, future clinical trials should consider provision of problem led, benign, and parsimonious interventions that target presenting difficulties (be they anxiety disorders, depression, or distressing attenuated psychotic experiences) rather than prevention of transition to first episode psychosis in itself.

This study has several clinical implications. Cognitive therapy reduces the severity of psychotic experiences without the use of antipsychotic drugs, which should provide the benefits of symptom based improvement without the associated risks of serious side effects. However, the low rate of transition to psychosis in both groups and the recovery from psychosis and affective symptoms is clearly an important and optimistic message to convey; both for young help seeking people meeting the criteria for being in an at risk mental state and for clinicians in contact with this population. It should encourage a normalising, non-catastrophic perspective on their psychotic experiences, and any treatment should largely be needs driven on the basis of what problems are presented and prioritised by service users; this is something that cognitive therapy is suited to, given its collaborative, problem oriented nature. However, active monitoring may also be beneficial and would be benign, easy to implement, and consistent with guidelines from the International Early Psychosis Association on treatment in the at risk phase $\mathrm{e}^{48}$; a period of watchful waiting involving regular monitoring that lasts for at least 12 months could be safely recommended. The ethics of intervening before the onset of a disorder are always debatable, and this is especially so in relation to the use of antipsychotics for people in an at risk mental state, which often have significant adverse effects; for example, a recent systematic review concluded that some of the structural abnormalities in brain volume that have previously been attributed to the syndrome of schizophrenia may be the result of antipsychotic drugs, ${ }^{49}$ which has been recently supported by a high quality prospective study ${ }^{50}$ as well as experimental studies in healthy volunteers. ${ }^{51}$ There is also evidence that increased cardiovascular risk is detectable after first exposure to any 
antipsychotic drug ${ }^{52}$ and there is overwhelming evidence of weight gain induced by antipsychotics. ${ }^{53}$ On the basis of low transition rates, high responsiveness to simple interventions such as monitoring, a specific effect of cognitive therapy on the severity of psychotic symptoms, and the toxicity associated with antipsychotic drugs, we would suggest that antipsychotics are not delivered as a first line treatment to people meeting the criteria for being in an at risk mental state.

We thank Hannah Taylor, Carolyn Crane, Melissa Wardle, and the other EDIE-2 (early detection and intervention evaluation for people at risk of psychosis) research assistants for their data collection work; our trial therapists (Jamie Kirk, Vicky Brooks, Rosalyn Hartwell, Aoiffe Kilcommons, Katie Mackie, Elizabeth Newton, and Gary Willington); the members of the trial steering committee (David Kingdon, Julia Renton, Paul Salkovskis, and Jo Smith) and the data monitoring and ethics committee (Douglas Turkington, Daniel Freeman, and Sabine Landau). Graham Dunn had full access to all of the data in the study and takes responsibility for the integrity of the data and the accuracy of the data analysis. We thank the Mental Health Research Network, the Scottish Mental Health Research Network, and the OpenCDMS team for their support and assistance. MB and PP were funded by the National Institute for Health Research through the Collaborations for Leadership in Applied Health Research and Care for Birmingham and Black Country (CLAHRC-BBC) programme. The views expressed in this publication are not necessarily those of the Medical Research Council, National Institute for Health Research, or Department of Health.

Contributors: All authors were involved in the design of the study and the ongoing management and delivery of the trial, and contributed to drafts of this manuscript. APM, the chief investigator, conceived the study, prepared the protocol, contributed to the training and supervision of the therapists and supervision of the researchers, had overall responsibility for the day to day running of the study, interpreted the data, and took the lead on writing the report. He is the guarantor for the study. PF, MB, DF, AIG, KB, JC, SP, and TR participated in preparation of the treatment protocol and the training and supervision of the therapists. MB, DF, AIG, and PBJ managed the additional sites. PF, AIG, PBJ, SWL, GKM, and PP trained the researchers in the psychiatric interviews and supervised and monitored standards of psychiatric interviewing and assessment throughout the trial. RPB, PBJ, SWL, and GKM also advised on diagnostic ratings and exclusions. SLKS was the trial manager. She supervised and coordinated recruitment, contributed to training of research staff, and was responsible for staff management and overall coordination of the study. GD was the trial statistician. He advised on randomisation and all statistical aspects of the trial, developed the analysis plan, and carried out the statistical analyses and is guarantor in this respect. LMD was the trial health economist. RB was a service user consultant involved in all aspects of the study.

Funding: This research was supported by the Medical Research Council (G0500264) and the Department of Health.

Competing interests: All authors have completed the ICMJE uniform disclosure form at www.icmje.org/coi_disclosure.pdf (available on request from the corresponding author) and declare: no support from any organisation for the submitted work; no financial relationships with any organisations that might have an interest in the submitted work in the previous three years; and no other relationships or activities that could appear to have influenced the submitted work.

Ethical approval: Our protocol was approved by the National Research Ethics Service of the UK National Health Service (NHS Eastern MREC 05/MRE05/61).

Data sharing: No additional data available.

Yung AR, McGorry PD, McFarlane C, Jackson HJ, Patton GC, Rakkar A. Monitoring and care of young people at incipient risk of psychosis. Schizoph Bull 1996;22:283-303.

2 McGorry PD, Yung AR, Phillips LJ, Yuen HP, Francey S, Cosgrave EM, et al. Randomized controlled trial of interventions designed to reduce the risk of progression to first-episode psychosis in a clinical sample with subthreshold symptoms. Arch Gen Psychiatry 2002;59:921-8.

3 Yung AR, Phillips LJ, Nelson B, Francey SM, PanYuen H, Simmons MB, et al. Randomized controlled trial of interventions for young people at ultra high risk for psychosis: 6-month analysis. J Clin Psychiatry 2011;72:430-40.

4 McGlashan TH, Zipursky RB, Perkins D, Addington J, Miller T, Woods SW, et al. Randomized, double-blind trial of olanzapine versus placebo in patients prodromally symptomatic for psychosis. Am J Psychiatry 2006;163:790-9.

5 Addington J, Epstein I, Liu L, French P, Boydell KM, Zipursky RB. A randomized controlled trial of cognitive behavioral therapy for individuals at clinical high risk of psychosis. Schizoph Res 2011;125:54-61.

6 Morrison AP, French P, Walford L, Lewis SW, Kilcommons A, Green J, et al. Cognitive therapy for the prevention of psychosis in people at ultra-high risk: randomised controlled trial. Br J Psychiatry 2004;185:291-7.

7 Morrison AP, French P, Parker S, Roberts M, Stevens H, Bentall RP, et al. 3 year follow-up of a randomised controlled trial of cognitive therapy for the prevention of psychosis in people at ultra-high risk. Schizoph Bull 2007;33:682-7.

8 Amminger GP, Schafer MR, Papageorgiou K, Klier CM, Cotton SM, Harrigan SM, et al. Long-chain omega- 3 fatty acids for indicated prevention of psychotic disorders: a randomized, placebo-controlled trial. Arch Gen Psychiatry 2010;67:146-54.

9 Morrison AP, Bentall RP, French P, Walford L, Kilcommons A, Knight A, et al. A randomised controlled trial of early detection and cognitive therapy for preventing transition to psychosis in high risk individuals: study design and interim analysis of transition rate and psychological risk factors. Br J Psychiatry 2002;181(suppl 43):78-84 .

10 Cognitive behaviour therapy for acute schizophrenia: a controlled trial. British Psychological Society Annual Conference; 1994; Brighton, UK.

11 Tarrier N, Yusupoff L, Kinner C, McCarthy E, Gladhill A, Haddock G, et al. A randomized controlled trial of intense cognitive behaviour therapy for chronic schizophrenia. $B M J$ 1998;317:303-7.

12 Sensky T, Turkington D, Kingdon D, Scott JL, Scott J, Siddle R, et al. A randomized controlled trial of cognitive-behavioral therapy for persistent symptoms in schizophrenia resistant to medication. Arch Gen Psychiatry 2000;57:165-72.

13 Birchwood M. Early intervention in psychotic relapse: cognitive approaches to detection and management. In: Haddock G, Slade PD, eds. Cognitive-behavioural interventions with psychotic disorders. Routledge, 1996:171-211.

14 Clark DM, Salkovskis PM, Hackmann A, Middleton H, Anastasiades P, Gelder M. A comparison of cognitive therapy, applied relaxation and imipramine in the treatment of panic disorder. Br J Psychiatry 1994;164:759-69.

15 Yung AR, Yuen HP, McGorry PD, Phillips LJ, Kelly D, Dell'Olio M, et al. Mapping the onset of psychosis-the Comprehensive Assessment of At Risk Mental States (CAARMS). Aust N Z J Psychiatry 2005;39:964-71

16 Morrison AP. The interpretation of intrusions in psychosis: an integrative cognitive approach to hallucinations and delusions. Behav Cogn Psychother 2001;29:257-76.

17 French P, Morrison AP. Early detection and cognitive therapy for people at high risk of developing psychosis: a treatment approach. Wiley, 2004.

18 Blackburn IM, James I, Milne D, Baker CA, Standart S, Garland A, et al. The revised cognitive therapy scale (CTS-R): psychometric properties. Behav Cogn Psychother 2001;29:431-46.

19 Bell K, Startup M, French P, Morrison AP, Bucci SS, Fowler D. Therapist treatment adherence in $\mathrm{CBT}$ for people at ultra-high risk for psychosis: development of a new rating scale. Early Interv Psychiatry 2008:2:16.

20 Beck AT, Guth D, Steer RA, Ball R. Screening for major depression disorders in medical inpatients with the Beck Depression Inventory for Primary Care. Behav Res Ther 1997;35:785-91.

21 Mattick RP, Clarke JC. Development and validation of measures of social phobia scrutiny fear and social interaction anxiety. Behav Res Ther 1998;36:455-70.

22 Priebe S, Huxley P, Knight S, Evans S. Application and results of the Manchester Short Assessment of Quality of Life (MANSA). Int J Soc Psychiatry 1999;45:7-12.

23 Spitzer RL, Williams JB, Gibbon M, First MB. The structured clinical interview for DSM-III-R (SCID): history, validation, and description. Arch Gen Psychiatry 1992;49:624-9.

24 Peters L. Discriminant validity of the Social Phobia and Anxiety Inventory (SPAI), the Social Phobia Scale (SPS) and the Social Interaction Anxiety Scale (SIAS). Behav Res Ther 2000;38:943-50.

25 Ainsworth JD, Harper RS. The PsyGrid experience: using web services in the study of schizophrenia. Int J Healthc Inf Syst Inform 2007;2:1-20.

26 Morrison AP, Stewart S, French P, Bentall RP, Birchwood M, Byrne RE, et al. Early Detection and Intervention Evaluation for people at high-risk of psychosis-2 (EDIE-2): trial rationale, design and baseline characteristics. Early Interv Psychiatry 2011;5:24-32.

27 Everitt BS, Dunn G. Applied multivariate data analysis . Arnold, 1991

28 Little RJA, Rubin DB. Statistical analysis with missing data. Wiley, 2002.

29 Hausman JA. Specification tests in econometrics. Econometrica 1978;46:1251-71.

30 Nagelkerke N, Fidler V, Bersen R, Borgdorff M. Estimating treatment effects in randomized clinical trials in the presence of non-compliance. Stat Med 2000;19:1849-64.

31 Maracy M, Dunn G. Estimating dose-response effects in psychological treatment trials: the role of instrumental variables. Stat Methods Med Res 2011;20:191-215.

32 Yung AR, Yuen HP, Berger G, Francey S, Hung T-C, Nelson B, et al. Declining transition rate in ultra high risk (prodromal) services: dilution or reduction of risk? Schizophr Bull 2007;33:673-81.

33 Cuijpers P, van Straten A, Bohlmeijer E, Hollon SD, Andersson G. The effects of psychotherapy for adult depression are overestimated: a meta-analysis of study quality and effect size. Psychol Med 2010;40:211-23.

34 Wykes T, Steel C, Everitt B, Tarrier N. Cognitive behavior therapy for schizophrenia: effect sizes, clinical models, and methodological rigor. Schizophr Bull 2008;34:523-37.

35 Van Os J. Is there a continuum of psychotic experiences in the general population? Epidemiol Psichiatr Soc 2003;12:242-52.

36 McGorry PD, Hickie IB, Yung AR, Pantelis C, Jackson HJ. Clinical staging of psychiatric disorders: a heuristic framework for choosing earlier, safer and more effective interventions. Aust N Z J Psychiatry 2006:40:616-22.

37 Ruhrmann S, Schultze-Lutter F, Salokangas RKR, Heinimaa M, Linszen D, Dingemans $\mathrm{P}$, et al. Prediction of psychosis in adolescents and young adults at high risk: results from the prospective European prediction of psychosis study. Arch Gen Psychiatry 2010;67:241-51.

38 Cannon TD, Cadenhead K, Cornblatt B, Woods SW, Addington J, Walker E, et al. Prediction of psychosis in youth at high clinical risk: a multisite longitudinal study in North America. Arch Gen Psychiatry 2008;65:28-37. 


\section{What is already known on this topic}

It seems possible to identify a population at ultra high risk of developing psychosis

Several small trials suggest promising interventions for the prevention of psychosis and improvement in psychotic symptoms in these populations

\section{What this study adds}

Cognitive therapy did not prevent transition to psychosis but did reduce the severity of psychotic symptoms in young people (aged 14-35) at risk of psychosis

The rates of transition are lower than previously thought and there is a high potential for recovery with minimal intervention in this population

It is important to reconsider whether young people meeting these criteria can be accurately described as being at "ultra high risk of psychosis"

39 Van Os J, Linscott RJ, Myin-Germeys I, Delespaul P, Krabbendam L. A systematic review and meta-analysis of the psychosis continuum: evidence for a psychosis proneness?persistence?impairment model of psychotic disorder. Psychol Med 2009;39:179-95.

40 Loewy RL, Johnson JK, Cannon TD. Self-report of attenuated psychotic experiences in a college population. Schizophr Res 2007;93:144-51.

41 Morrison AP, Byrne RE, Bentall RP. DSM-5 and the 'psychosis risk syndrome': whose best interests. would it serve? Psychosis: Psychol, Soc Integr Approaches 2010;2:96-9.

42 Varghese D, Scott J, Welham J, Bor W, Najman J, O'Callaghan M, et al. Psychotic-like experiences in major depression and anxiety disorders: a population-based survey in young adults. Schizophr Bull 2011:37:389-93.

43 Van Rossum I, Dominguez MD, Lieb R, Wittchen HU, van Os J. Affective dysregulation and reality distortion: a 10 -year prospective study of their association and clinical relevance. Schizophr Bull 2011;37:561-71.

44 Dominguez MD, Wichers M, Lieb R, Wittchen H-U, van Os J. Evidence that onset of clinical psychosis is an outcome of progressively more persistent subclinical psychotic experiences: an 8-year cohort study. Schizophr Bull 2011:37:84-93.

45 Foster C, Startup H, Potts L, Freeman D. A randomised controlled trial of a worry intervention for individuals with persistent persecutory delusions. J Behav Ther Exp Psychiatry 2010;41:45-51.

46 Addington J, Cornblatt BA, Cadenhead KS, Cannon TD, McGlashan TH, Perkins DO, et al. At clinical high risk for psychosis: outcome for nonconverters. Am J Psychiatry 2011;168:800-5.

47 Velthorst E, Nieman DH, Becker HE, van de Fliert R, Dingemans PM, Klaassen R, et al. Baseline differences in clinical symptomatology between ultra high risk subjects with and without a transition to psychosis. Schizophr Res 2009;109:60-5.

48 International Early Psychosis Association. International clinical practice guidelines for early psychosis. BrJ Psychiatry, 2005;(suppl)120-4.
49 Moncrieff J, Leo J. A systematic review of the effects of antipsychotic drugs on brain volume. Psychol Med 2010;40:1409-22.

50 Ho BC, Andreasen NC, Ziebell S, Pierson R, Magnotta V. Long-term antipsychotic treatment and brain volumes: a longitudinal study of first-episode schizophrenia. Arch Gen Psychiatry 2011;68:128-37.

51 Tost H, Braus DF, Hakimi S, Ruf M, Vollmert C, Hohn F, et al. Acute D2 receptor blockade induces rapid, reversible remodeling in human cortical-striatal circuits. Nat Neurosci 2010;13:920-2.

52 Foley DL, Morley KI. Systematic review of early cardiometabolic outcomes of the first treated episode of psychosis. Arch Gen Psychiatry 2011;68:609-16.

53 Alvarez-Jimenez M, Hetrick SE, Gonzalez-Blanch C, Gleeson JF, McGorry PD. Non-pharmacological management of antipsychotic-induced weight gain: systematic review and meta-analysis of randomised controlled trials. Br J Psychiatry 2008;193:101-7.

54 Phillips LJ, McGorry PD, Yuen HP, Ward J, Donovan K, Kelly D, et al. Medium term follow-up of a randomized controlled trial of interventions for young people at ultra high risk of psychosis. Schizophr Res 2007:96:25-33.

\section{Accepted: 14 February 2012}

\section{Cite this as: BMJ 2012;344:e2233}

This is an open-access article distributed under the terms of the Creative Commons Attribution Non-commercial License, which permits use, distribution, and reproduction in any medium, provided the original work is properly cited, the use is non commercial and is otherwise in compliance with the license. See: http://creativecommons.org/licenses/by$\mathrm{nc} / 2.0 /$ and http://creativecommons.org/licenses/by-nc/2.0/legalcode. 


\section{Tables}

\begin{tabular}{|c|c|c|c|c|c|c|c|}
\hline Study & $\begin{array}{l}\text { No }(\% \\
\text { male) }\end{array}$ & $\begin{array}{l}\text { Age } \\
\text { range }\end{array}$ & Intervention groups & $\begin{array}{l}\text { Intervention/follow-up } \\
\text { periods }\end{array}$ & $\begin{array}{c}\text { Transition rates } \\
\text { (\%) }\end{array}$ & Outcomes & $\begin{array}{c}\text { Dropout rates } \\
(\%)\end{array}$ \\
\hline $\begin{array}{l}\text { McGorry et al } \\
(2002)^{254}\end{array}$ & $59(58)$ & $14-30$ & $\begin{array}{l}\text { Antipsychotic } \\
\text { drugs+cognitive behaviour } \\
\text { therapy } v \text { needs based } \\
\text { intervention }\end{array}$ & $\begin{array}{l}6 \text { months' intervention; } \\
12 \text { months' follow-up; } 48 \\
\text { months' follow-up }{ }^{54}\end{array}$ & $\begin{array}{c}10 \vee 36 \text { at } 6 \\
\text { months; } 19 v 36 \\
\text { at } 12 \text { months }\end{array}$ & $\begin{array}{l}\text { Significant difference in transition } \\
\text { rate at } 6 \text { months but not at } 12 \\
\text { months. Both groups improved on } \\
\text { symptoms, but no difference } \\
\text { between groups. No differences at } \\
48 \text { months }\end{array}$ & $\begin{array}{c}42 \text { from } \\
\text { antipsychotic } \\
\text { drugs+cognitive } \\
\text { behaviour } \\
\text { therapy }\end{array}$ \\
\hline $\begin{array}{l}\text { Morrison et al } \\
(2004)^{67}\end{array}$ & $58(69)$ & $14-36$ & $\begin{array}{l}\text { Cognitive behaviour therapy } \\
v \text { monthly monitoring plus } \\
\text { treatment as usual }\end{array}$ & $\begin{array}{l}6 \text { months' intervention; } \\
12 \text { months' follow-up; } 36 \\
\text { months' follow-up }\end{array}$ & $\begin{array}{l}6 v 22-30 \text { at } 12 \\
\text { months }\end{array}$ & $\begin{array}{l}\text { Significant difference in transition } \\
\text { rate (using all three definitions) and } \\
\text { symptoms at } 12 \text { months; only } \\
\text { difference at } 36 \text { months was in } \\
\text { prescription of antipsychotic drugs }\end{array}$ & $\begin{array}{l}13.5 \text { from } \\
\text { cognitive } \\
\text { behaviour } \\
\text { therapy }\end{array}$ \\
\hline $\begin{array}{l}\text { McGlashan et } \\
\text { al }(2006)^{4}\end{array}$ & $60(65)$ & $12-36$ & $\begin{array}{l}\text { Antipsychotic drugs } v \\
\text { placebo }\end{array}$ & $\begin{array}{l}12 \text { months' intervention; } \\
24 \text { months' follow-up }\end{array}$ & $\begin{array}{c}16.1 v 37.9 \text { at } 12 \\
\text { months }\end{array}$ & $\begin{array}{l}\text { No significant difference in transition } \\
\text { rates at } 12 \text { or } 24 \text { months. Significant } \\
\text { difference in symptoms at } 12 \\
\text { months in favour of antipsychotic } \\
\text { drugs }\end{array}$ & $54.8 v 34.5$ \\
\hline $\begin{array}{l}\text { Amminger et } \\
\text { al }(2010)^{8}\end{array}$ & $81(34)$ & $13-25$ & $\begin{array}{l}\text { Polyunsaturated fatty } \\
\text { acids+needs based } \\
\text { psychological intervention } v \\
\text { placebo+needs based } \\
\text { psychological intervention }\end{array}$ & $\begin{array}{l}3 \text { months' intervention; } \\
12 \text { months' follow-up }\end{array}$ & $\begin{array}{l}4.0 v 27.5 \text { at } 12 \\
\text { months }\end{array}$ & $\begin{array}{l}\text { Significant differences in transition } \\
\text { rate and symptoms at } 12 \text { months in } \\
\text { favour of polyunsaturated fatty acids }\end{array}$ & $7.3 v 5$ \\
\hline $\begin{array}{l}\text { Addington et } \\
\text { al }(2011)^{5}\end{array}$ & $51(71)$ & $14-30$ & $\begin{array}{l}\text { Cognitive behaviour therapy } \\
v \text { supportive psychotherapy }\end{array}$ & $\begin{array}{l}6 \text { months' intervention; } \\
18 \text { months' follow-up }\end{array}$ & $\begin{array}{c}0 \vee 12.5 \text { at } 6 \\
\text { months }\end{array}$ & $\begin{array}{l}\text { No significant difference in transition } \\
\text { rates. Both groups improved on } \\
\text { symptoms, but no difference } \\
\text { between groups }\end{array}$ & $29.6 v 20.8$ \\
\hline $\begin{array}{l}\text { Yung et al } \\
(2011)^{3}\end{array}$ & $115(39)$ & $14-30$ & $\begin{array}{l}\text { Cognitive behaviour } \\
\text { therapy+antipsychotic drugs } \\
v \text { cognitive behaviour } \\
\text { therapy+placebo } v \\
\text { supportive } \\
\text { psychotherapy+placebo }\end{array}$ & $\begin{array}{l}12 \text { months' intervention } \\
\text { (6 months' interim } \\
\text { analysis) }\end{array}$ & $\begin{array}{c}4.7 \vee 9.1 \vee 7.1 \text { at } \\
6 \text { months } \\
\text { (interim) }\end{array}$ & $\begin{array}{l}\text { No significant difference in transition } \\
\text { rates; all groups improved on } \\
\text { primary symptom measure (interim } \\
\text { analysis) }\end{array}$ & $16 \vee 20 v 21$ \\
\hline
\end{tabular}


Table 2| Referral sources for participants

\begin{tabular}{lc} 
Referral source & No (\%) \\
\hline Early intervention for psychosis service & $97(33.6)$ \\
\hline General practitioner & $32(11.1)$ \\
\hline Primary care mental health team & $28(9.7)$ \\
\hline Youth counselling service & $24(8.3)$ \\
\hline Secondary care community mental health team & $22(7.6)$ \\
\hline University counselling service & $19(6.6)$ \\
\hline Housing or homeless services & $16(4.6)$ \\
\hline Child and adolescent mental health services & $15(5.2)$ \\
\hline Substance misuse services & $11(3.8)$ \\
\hline Self referral & $6(2.1)$ \\
\hline Acute psychiatric inpatient unit & $5(1.7)$ \\
\hline Youth offending team & $4(1.4)$ \\
\hline Secondary care crisis and home treatment team & $3(1.0)$ \\
\hline Family member or carer & $2(0.7)$ \\
\hline Other & $4(1.4)$ \\
\hline
\end{tabular}


Table 3| Baseline characteristics of participants. Values are means (standard deviations) unless stated otherwise

\begin{tabular}{|c|c|c|c|}
\hline Characteristics & Whole sample ( $\mathrm{n}=\mathbf{2 8 8})$ & Cognitive therapy plus monitoring $(n=144)$ & Monitoring only ( $n=144)$ \\
\hline Age & $20.74(4.34), \mathrm{n}=288$ & $20.73(4.18), \mathrm{n}=144$ & $20.75(4.50), n=144$ \\
\hline Male:female & $180: 108$ & 89:55 & $91: 53$ \\
\hline CAARMS severity (summed, 0-144) & 38.44 (17.30), $n=286$ & $38.72(16.84), n=143$ & $38.15(17.80), n=143$ \\
\hline CAARMS distress (average, 0-100) & $42.61(20.03), n=264$ & 42.77 (20.51), $n=130$ & 42.45 (19.62), $n=134$ \\
\hline Global assessment of functioning & $51.06(10.60), n=288$ & $50.98(10.98), n=144$ & $51.15(10.25), n=144$ \\
\hline Beck depression inventory-primary care total & $9.73(4.48), n=258$ & $10.41(4.15), n=131$ & $9.02(4.70), n=127$ \\
\hline No (\%) above Beck depression inventory threshold & 229/258 (89) & 122/131 (93) & $107 / 127(84)$ \\
\hline Social interaction anxiety scale total & $41.18(16.98), n=234$ & 42.88 (16.92), $n=121$ & $39.36(16.93), n=113$ \\
\hline No (\%) above social interaction anxiety scale threshold & $141 / 234(60)$ & $76 / 121(63)$ & $65 / 113(58)$ \\
\hline MANSA total & 47.70 (10.10), $n=195$ & $46.33(9.60), n=99$ & $49.10(11.00), n=96$ \\
\hline
\end{tabular}

CAARMS=comprehensive assessment of at risk mental state; MANSA=Manchester short assessment of quality of life. 
Table 4 | Number of transitions to psychosis in each group (and cumulative total) at each assessment occasion and maximum number available at each occasion (owing to variable follow-up design)

\begin{tabular}{|c|c|c|c|c|c|c|c|c|c|c|c|c|}
\hline \multirow[b]{2}{*}{ Group } & \multicolumn{12}{|c|}{ Month of assessment } \\
\hline & 1 & 2 & 3 & 4 & 5 & 6 & 9 & 12 & 15 & 18 & 21 & 24 \\
\hline Monitoring (cumulative total) & 1 & $2(3)$ & $0(3)$ & $2(5)$ & $1(6)$ & $0(6)$ & $3(9)$ & $1(10)$ & $0(10)$ & $1(11)$ & $1(12)$ & $1(13)$ \\
\hline Cognitive therapy (cumulative total) & 2 & $1(3)$ & $1(4)$ & $1(5)$ & $1(6)$ & $0(6)$ & $1(7)$ & $0(7)$ & $0(7)$ & $1(8)$ & $0(8)$ & $2(10)$ \\
\hline Maximum No & 288 & 288 & 288 & 288 & 288 & 288 & 288 & 288 & 251 & 224 & 195 & 164 \\
\hline
\end{tabular}


Table 5| Results of primary and secondary outcome variables at 6, 12, and 24 months. Values are means (standard deviations) unless stated otherwise

\begin{tabular}{|c|c|c|c|c|c|c|}
\hline \multirow[b]{2}{*}{ Variable } & \multicolumn{2}{|c|}{6 months } & \multicolumn{2}{|c|}{12 months } & \multicolumn{2}{|c|}{24 months } \\
\hline & Cognitive therapy & Monitoring only & Cognitive therapy & Monitoring only & Cognitive therapy & Monitoring only \\
\hline \multicolumn{7}{|l|}{ CAARMS: } \\
\hline Severity & 17.89 (16.50), n=97 & 18.69 (19.34), $n=99$ & 14.88 (15.54), $n=95$ & $20.84(17.75), n=93$ & 13.94 (16.07), $n=34$ & $18.84(20.52), n=31$ \\
\hline Distress & 18.33 (20.67), n=91 & 17.84 (19.43), n=97 & $14.72(16.87), \mathrm{n}=92$ & $19.49(18.26), \mathrm{n}=91$ & $12.82(16.56), n=33$ & $17.72(21.31), n=29$ \\
\hline Global assessment of functioning & $59.30(16.21), \mathrm{n}=97$ & $61.61(15.04), n=98$ & 60.74 (16.69), $\mathrm{n}=95$ & 58.59 (16.23), $\mathrm{n}=94$ & $64.12(17.71), n=34$ & $60.19(16.88), n=31$ \\
\hline \multicolumn{7}{|l|}{$\begin{array}{l}\text { Beck depression inventory-primary } \\
\text { care: }\end{array}$} \\
\hline Total score & 5.60 (4.72), n=92 & $5.61(4.79), n=93$ & $5.41(5.12), n=93$ & $5.72(4.92), \mathrm{n}=90$ & 4.85 (4.49), $\mathrm{n}=33$ & $6.00(4.77), \mathrm{n}=28$ \\
\hline No (\%) with score >3 & $56(61)$ & $55(59)$ & $51(55)$ & $55(61)$ & $19(58)$ & $15(54)$ \\
\hline \multicolumn{7}{|l|}{ Social interaction anxiety scale: } \\
\hline Total score & 30.85 (17.98), $n=82$ & $30.60(16.91), n=90$ & $32.51(17.08), n=91$ & $29.99(16.60), n=87$ & $27.81(17.30), n=32$ & $36.42(19.48), n=26$ \\
\hline No (\%) with score $>36$ & $30(37)$ & $36(40)$ & $37(41)$ & $32(37)$ & $9(28)$ & $11(42)$ \\
\hline MANSA & $53.17(12.38), n=75$ & $53.00(11.45), n=66$ & 53.38 (12.60), $\mathrm{n}=66$ & 54.65 (11.75), $n=68$ & $56.78(10.55), n=27$ & 52.33 (11.32), $\mathrm{n}=21$ \\
\hline
\end{tabular}




\section{Figure}

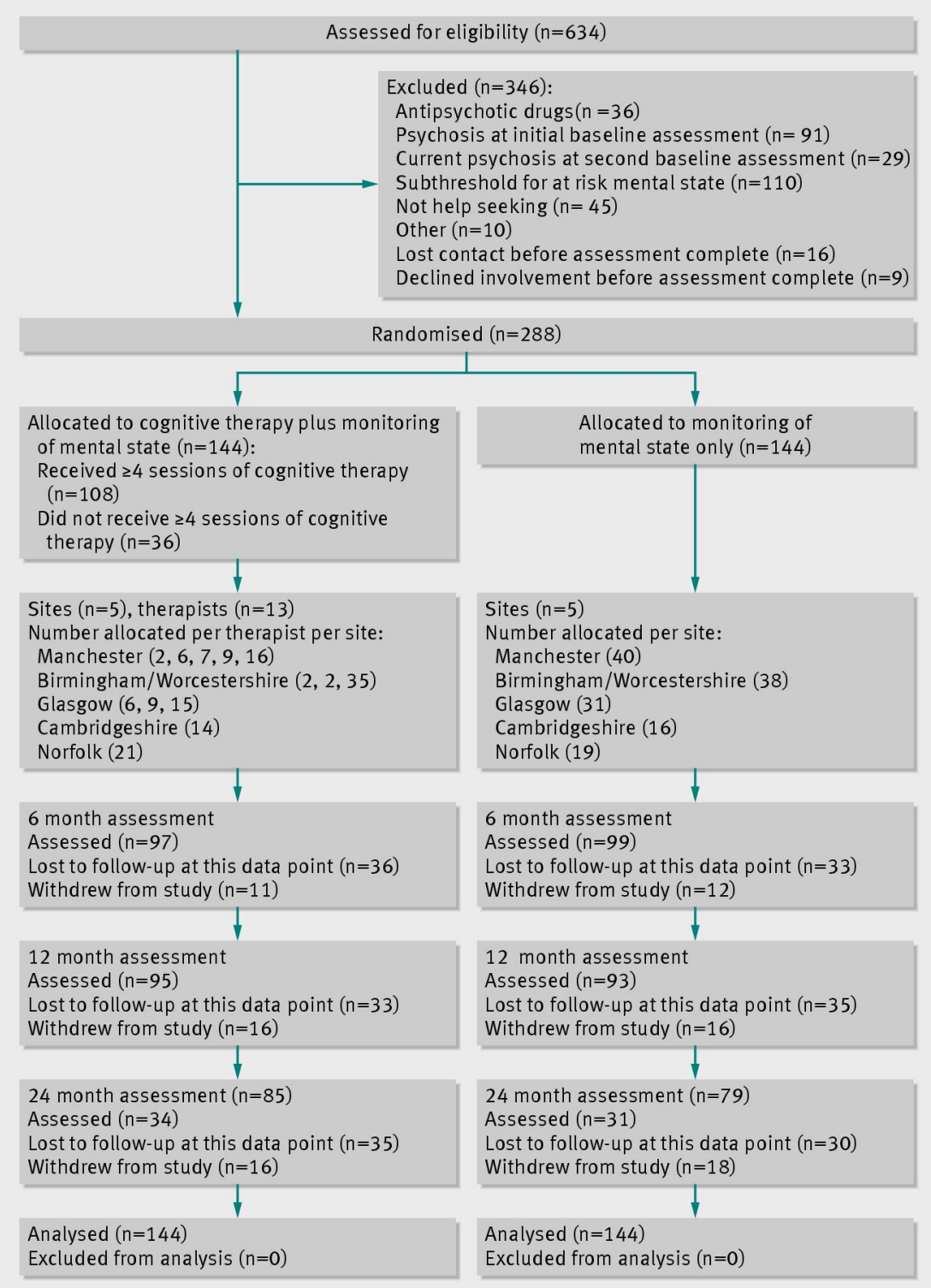

Flow of participants through study 